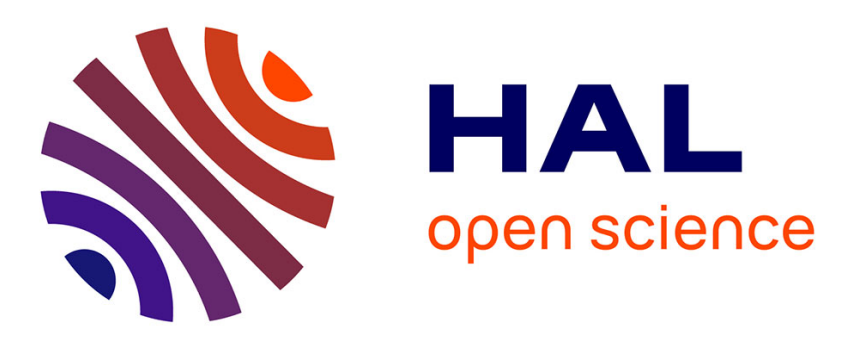

\title{
Two Modes of Sensorimotor Integration in Intention-Based and Stimulus-Based Actions
}

Arvid Herwig, Wolfgang Prinz, Florian Waszak

\section{To cite this version:}

Arvid Herwig, Wolfgang Prinz, Florian Waszak. Two Modes of Sensorimotor Integration in IntentionBased and Stimulus-Based Actions. Quarterly Journal of Experimental Psychology, 2007, 60 (11), pp.1540-1554. 10.1080/17470210601119134 . hal-02386093

\section{HAL Id: hal-02386093 https://hal.science/hal-02386093}

Submitted on 2 Dec 2019

HAL is a multi-disciplinary open access archive for the deposit and dissemination of scientific research documents, whether they are published or not. The documents may come from teaching and research institutions in France or abroad, or from public or private research centers.
L'archive ouverte pluridisciplinaire HAL, est destinée au dépôt et à la diffusion de documents scientifiques de niveau recherche, publiés ou non, émanant des établissements d'enseignement et de recherche français ou étrangers, des laboratoires publics ou privés. 
See discussions, stats, and author profiles for this publication at: https://www.researchgate.net/publication/259703523

\section{Two modes of sensorimotor integration in intention-based and stimulus-based actions}

Article in Quarterly journal of experimental psychology (2006) · December 2007

DOI: $10.1080 / 17470210601119134$

CITATIONS

137

3 authors, including:

Arvid Herwig

Universität Bremen

37 PUBLICATIONS 747 CITATIONS

SEE PROFILE
READS

86

Florian Waszak

Université de Paris

133 PUBLICATIONS 3,183 CITATIONS

SEE PROFILE

Some of the authors of this publication are also working on these related projects:

Project instruction-based action control View project 


\title{
Two modes of sensorimotor integration in intention-based and stimulus-based actions
}

\author{
Arvid Herwig and Wolfgang Prinz \\ Max Planck Institute for Human Cognitive and Brain Sciences, Leipzig, Germany \\ Florian Waszak \\ Laboratoire Psychologie de la Perception, CNRS \& Université René Descartes, Paris, France
}

\begin{abstract}
Human actions may be driven endogenously (to produce desired environmental effects) or exogenously (to accommodate to environmental demands). There is a large body of evidence indicating that these two kinds of action are controlled by different neural substrates. However, only little is known about what happens-in functional terms- on these different "routes to action". Ideomotor approaches claim that actions are selected with respect to their perceptual consequences. We report experiments that support the validity of the ideomotor principle and that, at the same time, show that it is subject to a far-reaching constraint: It holds for endogenously driven actions only! Our results suggest that the activity of the two "routes to action" is based on different types of learning: The activity of the system guiding stimulus-based actions is accompanied by stimulus-response (sensorimotor) learning, whereas the activity of the system controlling intention-based actions results in action-effect (ideomotor) learning.
\end{abstract}

There are two principal ways in which humans can interact with their environment. They may either carry out movements to manipulate the environment in order to produce desired environmental effects, or carry out movements to accommodate environmental demands. The first kind of movement may be referred to as voluntary, operant, or intentional. This kind of motor act is selected on the basis of the agent's intentions. The second kind is performed on the basis of prior stimulus events and may be called reaction.

\section{Neuroanatomical routes to action}

The question as to how humans' interactions with the environment are controlled is a key question in neuroscience. The past decades witnessed a great deal of research trying to understand the neural mechanisms underlying reactive and intentional actions. It has been shown that the two kinds of action are controlled by different neural substrates, even if the way intention-based and stimulusbased actions are manifested by the body are the same in terms of muscle activity, observed kinematics, and dynamics. Recent neuroscientific studies suggest that intention-based actions are mediated by fronto-striatal circuits, including the dorso-lateral prefrontal cortex, anterior cingulate, and SMA (medial premotor cortex). By contrast, stimulus-based actions are supposed to be controlled by different, task-dependent pathways (Passingham, Toni, \& Rushworth, 2000; Toni,

Correspondence should be addressed to Florian Waszak, Laboratoire Psychologie de la Perception, CNRS-Université Paris 5, UFR Biomédicale des Saints Pères, 45 rue des Sts Pères, 75270 Paris cedex 06, France. E-mail: f.waszak@gmx.net 
Rushworth, \& Passingham, 2001): one stream for movements directed to the location of a stimulus (including portions of the parietal cortex and of the ventral premotor cortex) and another stream (involving the ventral stream extending into the ventral prefrontal cortex) that is supposed to take effect whenever visual stimuli determine which among different possible actions is appropriate to perform (arbitrary mappings).

\section{Sensorimotor versus ideomotor learning}

Despite the steadily growing body of knowledge about the neuroanatomical substrate guiding the various kinds of action, only little is known about what happens-in functional terms-on these different "routes to action". With regard to stimulus-based actions, there are several general accounts. Most early and some modern accounts of reactive movements refer to some higher entity that intercedes between stimulus and response-the will (e.g., Donders, 1868/1969); “supervisory attentional system” (e.g., Norman \& Shallice, 1986). However, following Exner (1879), Hommel (2000) suggested recently that the cognitive system is prepared ahead of the stimulus to act upon presentation of a particular stimulus in a specified way. In this sense, reactions are like "prepared reflexes", which include a representation of the action to be performed and also a specification of the condition(s) under which the action should be carried out. It is assumed that a simple association process mediates the application/build-up of these stimulusresponse rules: Selecting (and executing) an action in response to a given stimulus creates a representation that "binds" the codes of the actionrelevant stimulus attributes and the corresponding action codes (stimulus-response bindings). These rules may be stored in memory, such that they may serve to trigger actions on subsequent occasions (see Allport, 1987; Logan, 1988; and also Hommel, Pösse, \& Waszak, 2000). A prominent example of the activity of these stimulus - response rules is "utilization behaviour" (Lhermitte, 1983; Shallice, Burgess, Schon, \& Baxter, 1989), a pathological failure of executive control that is characterized by inability to inhibit actions from being triggered by the mere sight of an object with which the action is habitually associated.

Evidently, exogenously driven actions exist on a continuum with regard to their "reflex-likeness". For real reflexes stimulus information is both a necessary and a sufficient condition, so that the execution of the action follows the presentation of the stimulus instantaneously, reflex-like. For other stimulus-based actions the stimulus may only be one source of information. The other is an intentional set to respond to that information in a particular way (which, in experimental settings, is usually specified through instructions). We assume that the more complex or time consuming these additional processing steps specified in the intentional set are, the less reflex-like the action. One possibility to decrease the reflexlikeness of exogenously driven actions is to decouple response selection and response execution by delaying the latter. For reasons outlined below, Experiments 2, 3a, and 3b do not only investigate reflex-like, immediate stimulus-based actions but also delayed stimulus-based actions.

As for intention-based actions, most scientific definitions consider an action to be voluntary if it aims at producing some internally prespecified effect. More importantly, action effects are also believed to play a core role in the control of intentional actions (see, Hommel, 2000; Prinz, 1997). The ideomotor theory of action control that can be traced back to the middle of the 19th century (Harless, 1861; Lotze, 1852) suggests a model of how humans, given a particular goal, select a suitable action. Ideomotor approaches claim that performing an action leaves behind an association between the action's motor code and the sensory effects the action produces ("action-effect bindings"). These associations are bidirectional and, thus, can be used to retrieve an action by "anticipating" its effects. In other words, intention-based actions are selected with respect to their perceptual consequences (Prinz, 1997).

One of the key findings supporting the ideomotor principle comes from Elsner and Hommel (2001). They made subjects first work through an acquisition phase, in which a self-selected 
keypress was always followed by a certain tone (e.g., left keypress $\rightarrow$ high-pitch tone; right keypress $\rightarrow$ low-pitch tone). In a subsequent test phase, the same tones were presented as target stimuli in response to which subjects had to perform a speeded-choice response. In this test phase, an action was performed faster in response to the tone that the action had previously produced (in the example, low-pitch tone $\rightarrow$ right keypress) than to a tone that had been produced by the alternative action (in the example, high-pitch tone $\rightarrow$ right keypress). In accordance with the ideomotor principle, this result suggests that the perception of a learned sensory effect activates "backwardly" the action it is associated with: In the acquisition phase, subjects had acquired associations between the keypresses and the subsequent tones, which, in the test phase, influence the activation of the choice responses. Moreover, Elsner et al. (2002) showed that the mere perception of learned action effects result in activation of the rostral SMA proper, which is straightforward evidence that the SMA- which takes part in the "voluntary route to action"-is engaged in the cortical representation of the learned association between actions and effects.

In short, there is evidence that intention-based actions, on the one hand, and stimulus-based actions, on the other hand, are controlled by different neural pathways and that the activity of these pathways relies on different kinds of memory traces. The activity of the reaction system is based on the compilation of stimulus-response rules specifying which motor routines actionrelevant objects habitually require (sensorimotor learning). By contrast, the activity of the volitional system should be based on the compilation of action-effect rules specifying which action produces which effect (ideomotor learning). If this framework concerning the difference in perceptuomotor integration (stimulus-response vs. action-effect) between the two kinds of action holds true, action-effect integration as demonstrated by Elsner and Hommel (2001) should occur only if the subjects operate in the voluntary "mode" of movement-that is, if the actions are controlled by the voluntary action system.
Four experiments were conducted to test this prediction by replicating the experiments by Elsner and Hommel (2001) with different types of acquisition phase (manipulated between subjects). These experiments compared an intentionbased acquisition phase (corresponding to the one used by Elsner \& Hommel) with different types of stimulus-based acquisition phase. Subjects of the intention-based groups were required to freely select between two possible actions. In the stimulus-based groups, by contrast, the participants' actions were triggered by external stimulus events.

However, in all groups the actions were contingently followed by certain effect tones. That is, participants performed in all groups certain actions (keypresses with the index finger of the left or the right hand), and in all groups the actions were immediately followed by effect tones (e.g., left keypress $\rightarrow$ high-pitch tone; right keypress $\rightarrow$ low-pitch tone). The only difference between the groups was whether the actions were selected in an intention- or in a stimulus-based mode of movement. If ideomotor learning takes place only if the participants operate in the intention-based mode, then transfer effects as demonstrated by Elsner and Hommel (2001) should be observed only with intentionbased acquisition, but not with stimulus-based acquisition.

\section{EXPERIMENT 1}

Experiment 1 replicated the experiment by Elsner and Hommel (2001) with two different types of acquisition phase. The first type-the intentionbased acquisition-corresponds to the one used by Elsner and Hommel. In this group, participants were instructed to produce one of two keypresses depending on their own choice. In contrast, the second type of acquisition-the stimulus-based acquisition-was composed of stimulus-based actions - that is, the actions that participants performed were triggered by external stimulus events. We explored two types of stimulus-based actions (cf. Passingham, Toni, \& Rushworth, 2000; 
Toni, Rushworth, \& Passingham, 2001): actions with arbitrary and actions with spatially compatible stimulus-response mappings.

\section{Material and methods}

\section{Participants}

A total of 78 participants (ages $20-30$ years) naïve to the purpose of the experiment participated in the study. The participants were randomly assigned to three groups of 26 participants each. For the first group of participants, the acquisition phase was intention based (INT). In the other two groups, the acquisition phase was stimulus based (spatial, stim-SPA and arbitrary, stim-ARB, see below). The data of 4 participants were lost due to technical failure.

\section{Stimuli and apparatus}

The display and timing were controlled by an IBM-compatible computer, interfaced to a 17inch EIZO FLEXSCAN 9080i-M monitor. The visual stimuli were displayed on a black background. A small white cross (+) presented in the centre of the screen served as fixation point. In the INT group, a white asterisk was presented about $0.8^{\circ}$ below the fixation point to signal the participant to choose an action. In the stim-SPA group, a small square was presented about $0.8^{\circ}$ below and about $0.8^{\circ}$ to the left or to the right of the fixation point to indicate the response. In the stim-ARB group, the response was indicated by a small green or red square presented about $0.8^{\circ}$ below the fixation point. The viewing distance was about $70 \mathrm{~cm}$. The asterisk and the squares subtended a visual angle of $0.3^{\circ}$ in width and $0.3^{\circ}$ in height. The participants performed the (stimulus-based and intention-based) actions with their left and right index fingers. Auditory stimuli were MIDI tones (instrument Marimba) of $400 \mathrm{~Hz}$ (low pitch) or $800 \mathrm{~Hz}$ (high pitch), presented simultaneously through the left and right speaker of a headphone. Both tones were $200 \mathrm{~ms}$ in duration.

\section{Procedure}

The experiment consisted of a single session of about 30 min and was divided into an acquisition phase and a test phase.

Acquisition phase, intention-based (INT group). On appearance of the asterisk, participants had to press one of the two response keys. The participants were instructed to choose the keypress at random "as if they tossed a coin before each trial". They were not told to respond as fast as possible.

Acquisition phase, stimulus-based (stim-SPA and stim- $A R B$ group). In the stimulus-based conditions the participants were required to press the key that corresponded to the target stimulus. In the stimSPA group, the actions were triggered by stimuli spatially compatible to the required responses (left square $\rightarrow$ left response, right square $\rightarrow$ right response); in the stim-ARB group, they were triggered by arbitrary stimuli (green square $\rightarrow$ left response; red square $\rightarrow$ right response; counterbalanced across subjects). Participants were instructed to respond as fast as possible. Responses were given on a keyboard with two response keys separated by a horizontal distance of $13.5 \mathrm{~mm}$.

In all three groups, each keypress (left/right) triggered a particular effect tone (high/low). The tone was presented immediately after the keypress (stimulus onset asynchrony $=0 \mathrm{~ms}$ ). The actioneffect mapping was balanced across subjects.

Incorrect keypresses, response omissions, and anticipations were recorded and fed back to the participants by a visual warning message. After the response and the effect tone, the display went blank for $1,000 \mathrm{~ms}$ (except for the fixation point). After that, the procedure was repeated for the next trial. Participants worked through 20 practice trials and 260 acquisition trials (i.e., two action-effect pairs with 130 repetitions each).

Test phase. The test phase was the same for all three groups of participants. After completing the acquisition trials, participants were instructed for the test phase. In each test trial, one of the two effect tones was presented as a target stimulus in response to which participants were required to 
respond as fast as possible according to a fixed stimulus-response mapping.

For each of the three acquisition groups, there were two subgroups of participants: In the acquisition-compatible subgroup, the participants had to respond with the key that preceded the tone in the acquisition phase. For instance, participants who had experienced the action-effect mapping "left key $\rightarrow$ low tone/right key $\rightarrow$ high tone" were now required to respond to the low tone with the left index finger and to the high tone with the right index finger. In the acquisitionincompatible groups, the participants were to respond with the key that preceded the other tone in the acquisition phase. For instance, participants who had experienced the action-effect mapping "left key $\rightarrow$ low tone/right key $\rightarrow$ high tone" were now required to respond to the low tone with the right index finger and to the high tone with the left index finger.

Each test trial started after an intertrial interval (ITI) of 1,000 ms. After the ITI the low or high tone was presented for $200 \mathrm{~ms}$. The next ITI started immediately after the participants' reaction. Incorrect keypresses, response omissions, and anticipations were treated as in the acquisition phase. Participants worked through 10 practice trials and 200 valid test trials (two effect-response mappings with 100 repetitions each).

\section{Results}

The significance criterion was set to $p<.05$ for all analyses (one-tailed for $t$ tests of predicted effects).

\section{Acquisition phase}

We did not analyse RTs in the intention-based group, since the reactions in this group were not speeded. Suffice it to say that participants executed freely selected actions about $220 \mathrm{~ms}$ after the onset of the asterisk.

For the stimulus-based groups, only correct responses were analysed (stim-SPA: 99.1\%; stimARB: 96.7\%). Individual median reaction times (RTs) were calculated. Not surprisingly, spatially compatible reactions (stim-SPA overall: $273 \mathrm{~ms}$ ) were faster than arbitrary reactions (stim-ARB overall: $387 \mathrm{~ms}$ ). Importantly, within the two stimulus-based groups (i.e., between the acquisition-compatible vs. acquisition-incompatible subgroups), RTs in the acquisition phase were not different.

\section{Test phase}

Only correct responses were analysed (stim-SPA: 96.2\%; stim-ARB: 97.5\%; INT: 94.9\%). Table 1 shows median RTs and percentages of error as a function of acquisition group (INT vs. stim-SPA vs. stim-ARB) and compatibility (acquisition-compatible vs. acquisition-incompatible). The error pattern did not counteract the RT pattern; thus, a speed-accuracy trade-off can be excluded. The rightmost column of Table 1 shows the compatibility effect for the three acquisition groups: mean $\mathrm{RT}$ (incompatible)-mean RT(compatible). In the intention-based acquisition group (INT), mean RTs of participants responding with the "compatible" actions (i.e., the actions that preceded the target tones in the acquisition phase) were significantly shorter than mean RTs of participants responding with the "incompatible" actions (i.e., the actions that preceded the "other" tone in the acquisition phase). This result is a replication of the finding of Elsner and Hommel (e.g., 2001) described above. More importantly, in both stimulus-based acquisition groups (stim-SPA and stim-ARB), the pattern of result was reversed. The pattern of

Table 1. Reaction times and percentages of error in the test phase of Experiment 1, as a function of learning group and compatibility, and compatibility effect for reaction times

\begin{tabular}{lllll}
\hline Acquisition & Compatibility & $R T$ & $P E$ & $C E$ \\
\hline INT & compatible & $288(10)$ & 5.3 & $36^{*}$ \\
& incompatible & $324(15)$ & 4.3 & \\
Stim-ARB & compatible & $322(11)$ & 2.3 & $-31^{*}$ \\
& incompatible & $291(9)$ & 2.3 & \\
Stim-SPA & compatible & $308(16)$ & 4.8 & -30 \\
& incompatible & $278(10)$ & 2.5 & \\
\hline
\end{tabular}

Note: RT: reaction times (in ms; $S E$ in parentheses). PE: percentages of error (in \%). CE: compatibility effect (incompatible-compatible). INT: intention based. Stim: stimulus based. SPA: spatial. ARB: arbitrary.

${ }^{*} p<.05$. 
results is confirmed by an analysis of variance (ANOVA) with two between-subjects factors: 3 (acquisition group) $\times 2$ (compatibility group). The only significant effect was the interaction, $F(2,68)$ $=5.145, p<.01$, demonstrating that effect of compatibility differs between the acquisition groups. Separate $t$ tests revealed the compatibility effect to be significant (or almost significant) in all three acquisition groups: group INT, $t(24)=2.06$, $p<.05$; group stim-SPA, $t(22)=1.59, p=.06$; group stim-ARB, $t(22)=2.11, p<.05$.

\section{Discussion}

The results of Experiment 1 strongly support the hypothesis that action-effect learning depends on the mode of movement. Action-effect learning as demonstrated by Elsner and Hommel (2001) was found only for the intention-based acquisition group (INT). In this group an action was indeed faster if triggered by a tone that had previously been produced by the action (compatible condition) than if triggered by a tone that had been produced by the alternative action (incompatible condition).

In contrast to the intention-based acquisition group (INT), participants of both stimulus-based acquisition groups (stim-SPA and stim-ARB) were faster when they had to respond with the incompatible action than when they had to respond with the compatible action. The fact that the compatibility effect is negative could be explained by assuming that, in the course of each stimulus-response $(\mathrm{S}-\mathrm{R})$ event, an event file (Hommel, 1998) is compiled that does not only link action-relevant stimulus features with the corresponding action codes (e.g., Logan \& Etherton, 1994; Waszak, Hommel, \& Allport, 2003, 2005a), but that also includes the information that other stimuli presented during or directly after the stimulus-response event do not take part in the $\mathrm{S}-\mathrm{R}$ episode. These other stimulus events may be labelled with tags similar to the "to-beignored” tags (Neill, 1997; Neill \& Valdes, 1992) proposed to explain negative priming (for a review see, Fox, 1995). These tags may establish a kind of negative association between the code of the stimulus (action effect) and the code of the action so that presenting the action effects as targets interferes with the execution of an acquisition-compatible action. However, in all following experiments, we found a null effect with stimulus-based acquisition, as originally assumed. Therefore, we will not come back to this issue.

\section{EXPERIMENT 2}

Experiment 2 explored which of two factors boosts/hampers ideomotor learning. To do so, Experiment 2 contrasted stimulus-based arbitrary mappings that were executed either immediately after stimulus onset (immediate) or withheld until the presentation of a go signal (delayed). We suppose that delayed actions take an intermediate position between intention-based and immediate stimulus-based actions. On the one hand, the actions are selected with respect to some external information and, therefore, can be called stimulus based. On the other hand, the execution of the motor programme resembles intention-based actions in that the action is not carried out in a reflex-like fashion. Let us assume that ideomotor learning occurs only if the locus of response selection is internal (i.e., if the decision which action to perform is determined voluntarily) but that it breaks down whenever the locus of response selection is external (i.e., whenever the decision which action to perform is subject to an external constraint). If so, transfer effects as shown by Elsner and Hommel (2001) should be observed with intention-based acquisition only, but not with stimulus-based acquisition, whether delayed or immediate. If, by contrast, it is the "reflex-likeness" of response execution that matters (i.e., if ideomotor learning does not take place if actions are carried out in a speeded reflex-like manner), then one would expect to find transfer effects with intention-based as well as with delayed stimulus-based acquisition.

To replicate the effect of the intention-based acquisition group found in Experiment 1, Experiment 2 also included an intention-based acquisition group. 


\section{Method}

Participants

A sample of 83 participants (ages 21-37 years) participated in Experiment 2. The participants were randomly assigned to three groups with 24 participants each. For the first group of participants, the acquisition phase was intention based (INT). In the other two groups, the acquisition phase was stimulus based: stim-IMM (immediately) and stim-DEL (delayed), see below. In order to increase the statistical power for the contrast within the stim-IMM group we increased the number of participants of this group by 8 . The data of 3 participants were lost due to technical failure (2 stim-DEL; 1 stim-IMM).

\section{Material, methods, stimuli, and apparatus}

The methods of Experiment 2 were the same as those in Experiment 1, with some minor exceptions described in the following. (Most changes were done to make the experiment more similar to the original study of Elsner \& Hommel, 2001.) In the two stimulus-based groups (stimIMM and stim-DEL) either a green or a red asterisk ( $0.3^{\circ}$ in width and $0.3^{\circ}$ in height) was used to indicate to the participant which action to perform (instead of a square as in Experiment 1). In the stim-DEL group, a second white asterisk presented at the same position as the first coloured asterisk served as a go signal for the execution of the precued response.

\section{Procedure}

Acquisition phase. The acquisition phase of the INT and stim-IMM group was the same as that in Experiment 1. Participants in the new stimDEL group were required to withhold their response until presentation of the go signal, which appeared $800 \mathrm{~ms}$ after the onset of the coloured cue. Once again, in all groups, the participants' keypress (left/right) triggered a particular effect tone (high/low). However, just as in the original study of Elsner and Hommel (2001) the tone was now presented $50 \mathrm{~ms}$ after the keypress. As in the study from Elsner and Hommel (2001), erroneous trials were repeated at the end of the block, and participants worked now through 8 practice trials and 200 valid acquisition trials (Experiment 1: 260 trials) with an ITI of 1,500 ms (Experiment 1: 1,000 ms).

Test phase. The test phase was the same for all three groups of participants. The only changes to Experiment 1 concerned the ITI (now 1,500 ms instead of 1,000 ms).

\section{Results}

\section{Acquisition phase}

Participants of the intention-based group executed freely selected actions about $274 \mathrm{~ms}$ after the onset of the asterisk.

For the stimulus-based groups, only correct responses without omissions or anticipations were analysed (stim-IMM: 97.1\%; stim-DEL: 96.3\%). Since, in contrast to Experiment 1, trials with latencies exceeding 1,000 ms were excluded, individual mean RTs instead of median RTs were calculated. Precued reactions (stim-DEL overall: $280 \mathrm{~ms}$ ) were faster than uncued reactions (stim-IMM overall: $383 \mathrm{~ms}$ ). RTs did not differ between the acquisition-compatible versus acquisition-incompatible subgroups.

\section{Test phase}

Trials with response omissions (0.35 \%) and anticipations $(0.03 \%)$ were excluded from the analysis. Furthermore, only RTs from correct responses were analysed (INT: 98.2\%; stimIMM: 97.9\%; stim-DEL: 98.0\%). Table 2 shows mean RTs and percentages of error (PE) as a function of acquisition group (INT vs. stim-IMM vs. stim-DEL) and compatibility (acquisitioncompatible vs. acquisition-incompatible). An effect of compatibility was observed in the intention-based group only (INT $=71 \mathrm{~ms}$ ). In both stimulus-based acquisition groups there was no compatibility effect whatsoever (stim-IMM = $10 \mathrm{~ms}$; stim-DEL $=-8 \mathrm{~ms}$ ). An ANOVA was run including the two between-subjects factors acquisition group (INT vs. stim-IMM vs. stimDEL) and compatibility group (acquisitioncompatible vs. acquisition-incompatible). The 
Table 2. Reaction times and percentages of error in the test phase of Experiment 2, as a function of learning group and compatibility, and compatibility effect for reaction times

\begin{tabular}{llccr}
\hline Acquisition & Compatibility & $R T$ & $P E$ & $C E$ \\
\hline INT & compatible & $318(14)$ & $1.7(0.4)$ & $71^{*}$ \\
& incompatible & $389(15)$ & $2.0(0.7)$ & \\
Stim-IMM & compatible & $312(15)$ & $1.5(0.3)$ & 10 \\
& incompatible & $322(14)$ & $2.6(0.4)$ & \\
Stim-DEL & compatible & $347(18)$ & $1.1(0.4)$ & -8 \\
& incompatible & $339(18)$ & $2.9(0.8)$ & \\
\hline
\end{tabular}

Note: RT: reaction times (in ms; $S E$ in parentheses). PE: percentages of error (in \%). CE: compatibility effect (incompatible-compatible). INT: intention based. Stim: stimulus based. IMM: immediately. DEL: delayed.

$* p<.05$.

almost significant main effects of acquisition group, $F(2,74)=3.024, p=.06$, and compatibility group, $F(1,74)=3.517, p=.07$, were qualified by a significant interaction, $F(2,74)=3.119, p<$ .05 , demonstrating that effect of compatibility differs between the acquisition groups. Separate $t$ tests revealed the compatibility effect to be significant in the intention-based acquisition group: group INT, $t(22)=3.45, p<.01$, but not in the two stimulus-based acquisition groups: group stim-IMM, $t(30)=0.50, p=.31$; group stimDEL, $t(22)=-0.33, p=.37$.

Although incorrect keypresses were rare (between 1.1 and $2.9 \%$ ), an ANOVA of error rates revealed a significant main effect of compatibility group, $F(1,74)=6.684, p<.05$, which indicated fewer response errors of participants responding with the "compatible" actions than of participants responding with the "incompatible" actions. In view of the $\mathrm{PE}$ values reported in Table 2 this compatibility effect seems to be based primarily on the two stimulus-based acquisition groups. In other words, it looks as if there was some kind of speed-accuracy trade-off in the stimulus-based groups. Evidently this would undermine our line of argument (viz., that there was no compatibility effect in the stimulus-based acquisition groups). However, first of all, notice that neither the main effect of acquisition group nor the interaction was significant $\left(\right.$ all $F_{\mathrm{S}}<1$ ) for PEs. Second, neither the original study by Elsner and Hommel (2001) nor the other experiments of the present study (Experiments 1, 3a, and $3 \mathrm{~b}$ ) revealed a compatibility effect in the error rates, whatsoever. Hence, although the error data of Experiment 2 yielded a compatibility effect for all acquisition groups, in view of the whole data pattern of the present study, this result should not be overrated.

\section{Discussion}

The results of the RT analysis once again confirmed that action-effect learning depends on the mode of movement. In accordance with Experiment 1, a positive compatibility effect was found only for the INT group. By contrast, in the stimulus-based groups, no compatibility effect was observed at all. A more detailed discussion of the results is delayed until the General Discussion.

\section{EXPERIMENTS 3A AND 3B}

Evidently, it is possible that the stimulus-based acquisition groups fail to show compatibility effects due to lack of statistical power. To overcome this problem, we ran two additional experiments with larger sample sizes, which focused on the stimulus-based acquisition. In Experiments $3 \mathrm{a}$ and $3 \mathrm{~b}$ we systematically crossed the two factors investigated in the first two experiments: We explored stimulus-based actions that had either arbitrary or spatial mappings and that were executed either immediately or delayed.

\section{Method}

\section{Participants}

A sample of 160 participants (ages 19-37 years) participated in Experiment 3a (100 participants) and in Experiment 3b (60 participants). There were two stimulus-based acquisition groups in both experiments. Whereas in Experiment 3a participants performed either delayed (stim-DEL-ARB) or immediate (stim-IMM-ARB) actions in response 
to arbitrary stimuli, participants in Experiment 3b performed delayed (stim-DEL-SPA) or immediate (stim-IMM-SPA) actions in response to spatial stimuli. The participants of Experiment 3a were randomly assigned to the two groups with 40 participants each. In order to increase the statistical power for the contrast within the stim-IMM-ARB group, we increased the number of participants of this group by 20. The participants of Experiment 3b were randomly assigned to the two groups with 30 participants each.

Material, methods, stimuli, apparatus, and procedure The stim-IMM-ARB group (Experiment 3a) and the stim-IMM-SPA group (Experiment $3 b$ ) were replications of the stim-ARB and stim-SPA groups of Experiment 1. The stim-DEL-ARB (Experiment $3 \mathrm{a}$ ) resembled the stim-DEL group of Experiment 2 with the only exception that now squares instead of asterisks were used. The procedure of the stim-DEL-SPA was the same as the procedure of the stim-DEL-ARB group, except that spatial stimuli were used to cue the response. In this group, the go signal was presented just around the fixation cross.

\section{Results and discussion}

\section{Acquisition phase}

Only correct responses without omissions or anticipations were analysed (stim-IMM-ARB:
96.5\%; stim-DEL-ARB: 98.2\%; stim-IMMSPA: 99.1\%; stim-DEL-SPA: 99.1\%). Whereas precued reactions in Experiment 3a (stim-DELARB overall: $283 \mathrm{~ms}$ ) were faster than uncued reactions (stim-IMM-ARB overall: $388 \mathrm{~ms}$ ), this was not the case in Experiment 3b (stim-DELSPA overall: $304 \mathrm{~ms}$; stim-IMM-SPA overall: $290 \mathrm{~ms}$ ). This might well be due to a floor effect in the spatial groups. RTs in the acquisition phase were not different between the acquisitioncompatible versus acquisition-incompatible subgroups.

\section{Test phase}

Missed trials (0.04\% and 0.04\% in Experiments 3a and $3 \mathrm{~b}$, respectively) and anticipations $(0.53 \%$ and $0.52 \%$ ) were excluded from the analysis, and mean RTs and PEs were calculated and analysed analogously to Experiment 2. As can be seen in Table 3, there were no significant compatibility effects in Experiment 3a (stim-IMM-ARB $=6 \mathrm{~ms}$; stim$\mathrm{DEL}-\mathrm{ARB}=-6 \mathrm{~ms}$ ), as well as in Experiment 3b (stim-IMM-SPA $=7 \mathrm{~ms}$; stim-DEL-SPA = $-26 \mathrm{~ms})$. Neither the RT analysis nor the PE analysis yielded any significant effect (all $F_{\mathrm{S}}<$ $2.2, p>.15)$.

Experiments $3 \mathrm{a}$ and $3 \mathrm{~b}$ once again confirm that there is no action-effect integration as demonstrated by Elsner and Hommel (2001) if participants performed a stimulus-based acquisition phase. This was true for arbitrary and spatial as

Table 3. Reaction times and percentages of error in the test phase of Experiments $3 a$ and 3b, as a function of learning group and compatibility, and compatibility effect for reaction times

\begin{tabular}{|c|c|c|c|c|c|}
\hline Experiment & Acquisition & Compatibility & $R T$ & $P E$ & $C E$ \\
\hline \multirow[t]{4}{*}{$3 a(A R B)$} & \multirow[t]{2}{*}{ stim-IMM } & compatible & $314(9)$ & $2.4(0.4)$ & \multirow[t]{2}{*}{6} \\
\hline & & incompatible & $320(9)$ & $2.8(0.4)$ & \\
\hline & \multirow[t]{2}{*}{ stim-DEL } & compatible & $320(11)$ & $2.2(0.4)$ & \multirow[t]{2}{*}{-6} \\
\hline & & incompatible & 314 (14) & $2.3(0.4)$ & \\
\hline \multirow[t]{4}{*}{$3 \mathrm{~b}(\mathrm{SPA})$} & \multirow[t]{2}{*}{ stim-IMM } & compatible & $325(13)$ & $3.0(0.8)$ & \multirow[t]{2}{*}{7} \\
\hline & & incompatible & $332(17)$ & $3.0(0.8)$ & \\
\hline & \multirow[t]{2}{*}{ stim-DEL } & compatible & $353(15)$ & $2.8(0.5)$ & \multirow[t]{2}{*}{-26} \\
\hline & & incompatible & $327(8)$ & $1.7(0.3)$ & \\
\hline
\end{tabular}

Note: RT: reaction times (in ms; $S E$ in parentheses). PE: percentages of error (in \%). CE: compatibility effect (incompatible/ compatible). ARB: arbitrary stimulus-response mapping. SPA: spatial stimulus-response mapping. Stim: stimulus based. IMM: immediately. DEL: delayed. 
well as for immediate and delayed stimulus-based acquisition phases. To further corroborate this finding, we meta-analysed the data at hand by running an ANOVA on the RT data of Experiments 2, 3a, and $3 \mathrm{~b}$ with the betweensubject factors compatibility group (acquisitioncompatible vs. acquisition-incompatible) and experimental condition (including all in all six stimulus-based acquisition groups with a total of 216 participants). Neither the main effects nor the interactions were significant: main effect of compatibility group, $F(1,204)=0.718$; main effect of experimental condition, $F(5,204)=$ 1.509; interaction $F(5,204)=0.520$. A post hoc power analysis revealed that the sample size of 216 participants was sufficient to detect medium effect sizes following the conventions of Cohen $(1988 ; f=0.25)$ with a high power of .96 for the main effect of compatibility group when the conventional significance level of alpha $=.05$ is used (Faul \& Erdfelder, 1992). We believe that these data are sufficient to claim that-under the stimulus-based acquisition and the test conditions described above-there is no effect of the compatibility between action-effect mapping in the acquisition phase and stimulus-response mapping in the test phase.

\section{GENERAL DISCUSSION}

The starting point of the present study was the steadily growing body of evidence indicating that (one and the same) overt action is controlled by different neural substrates depending on whether the action is intention based or stimulus based. In the Introduction, we suggested that the activity of these different pathways is based on different types of memory traces - namely, that the activity of the system guiding stimulus-based actions is accompanied by stimulus-response (sensorimotor) learning, whereas the activity of the system controlling intention-based actions results in action-effect (ideomotor) learning. If this holds true, then acquisition-test transfer effects of action-effect learning as demonstrated by Elsner and Hommel (2001) should occur only if the actions in the acquisition phase are controlled by the voluntary action system.

The results strongly support this hypothesis. They back once more the validity of the ideomotor principle: Performing an action results in a bidirectional association between the motor patterns and ensuing sensory effects. These associations are then used backwardly in order to retrieve an appropriate action by "anticipating" desired action effects. However, the results also demonstrate that the ideomotor principle is subject to a far-reaching constraint: It holds for intentionbased actions only! It is only in the intentionbased acquisition group (INT) of Experiments 1 and 2 that an action was faster if triggered by a tone that the action had previously produced (compatible condition) than if triggered by a tone that had been produced by the alternative action (incompatible condition). In contrast, in the stimulus-based acquisition groups, there was either a negative compatibility effect (Experiment 1; for a discussion see Experiment 1) or no compatibility effect whatsoever (Experiments 2, 3a, and 3b). This result shows that the learning mechanism underlying the ideomotor principle is not as simple as originally assumed by Elsner and Hommel (2001; see also Elsner \& Hommel, 2004; Elsner et al., 2002). They proposed that action-effect learning has a very simple associative basis - namely, that whenever a particular action code and a particular sensory code are frequently coactivated, these codes are integrated automatically, regardless of the context the movement is carried out in.

In accordance with the results of the present study are recent findings from Astor-Jack and Haggard (2005). Astor-Jack and Haggard suggest that the motor system for internally generated actions and the system for externally triggered actions cannot be simultaneously active. They showed that preparing an intention-based action reduces the participants' "reactivity", in that the motor output system is shielded against being accessed from the stimulus-based system. The same may hold true the other way round-that is, preparing a stimulus-based action may shield the motor output system against being accessed 
from the intention-based system. The effect of this exclusivity of access to the motor system may be that an action is bound to the triggering stimulus or to the ensuing effect, respectively.

In a nutshell, we propose the following framework to account for the results presented above. In a stimulus-action-effect sequence, the codes of those two events, which belong to the given processing episode, are bound together. In the intention-based mode of movement, the memory trace associates action and ensuing effect. In the stimulus-based mode, however, the trace associates target stimulus and ensuing action. This is because the two modes work in a different fashion. In the stimulus-based mode, the participants pass on control to the stimuli in that the system merely acts upon presentation of a particular stimulus in a prespecified way (prepared reflex, Exner, 1879; Hommel, 2000). In this mode, actions are selected with respect to their sensory antecedents. In the intention-based mode, by contrast, actions are guided by the ideomotor principle-that is, they are selected with respect to their sensory consequences.

As concerns the intention-based mode, it could be questioned that the reported effect is indeed due to the compilation of bidirectional action-effect associations during the acquisition phase. One might argue that the effect in the intentional groups was based on the greater similarity between the acquisition phase and the test phase for this group. However, first of all notice that the assumption of one bidirectional association is widely accepted and was recently supported in a study with humans as well as with rats (Arcediano, Escobar, \& Miller, 2005). Second, several other studies very similar to the conditions with intention-based acquisition reported above already provided direct evidence for the assumption that, in the intention-based mode, bidirectional associations between action and effect are compiled and later on automatically retrieved. Elsner et al. (2002) showed that the mere perception of auditory stimuli that previously had been presented as effect tones of certain actions result in the activation of neural motor structures. Thus, action effects directly activate associated motor codes. Moreover, Kunde (2004) observed response priming by visual stimuli formerly presented as action effects although the effects were masked during the test phase and thus could not be consciously identified. Furthermore, unlike the present study, Kunde used a within-subject design. Kunde's results, therefore, rule out any strategic explanation or any explanation based on the similarity between acquisition and test phase.

As concerns the stimulus-based mode, one may object that ideomotor learning has already been demonstrated when participants operate in a "reactive" action control mode. Indeed, there is evidence for response-effect learning that has been obtained in experiments with "stimulusbased" actions (Beckers, De Houwer, \& Eelen, 2002; Elsner \& Hommel, 2004; Hommel, 1996, 2004; Hoffmann, Sebald, \& Stoecker, 2001; Kunde, Hoffmann \& Zellmann, 2002; Ziessler, 1998; Ziessler \& Nattkemper, 2001, 2002). At the moment, we do not have a complete understanding of the experimental parameters and boundary conditions that lead to the diverging patterns of results, but we see two possible reasons: First, as mentioned in the Introduction, exogenously driven actions exist on a continuum with regard to their "reflex-likeness", with real reflexes being driven by stimulus information as a necessary and sufficient condition and other stimulus-based actions depending on an intentional set that specifies how to respond to which stimulus. The more complex this intentional set, the less reflex-like the action. The immediate stimulusbased actions used in the present study were relatively easy or reflex-like compared to most of, if not all, the studies mentioned above. Beckers et al. (2002), for example, used word stimuli and categorical judgements. Ziessler and Nattkemper (2001) used a serial reaction task with eight possible stimuli. In the study of Kunde et al. (2002), participants had to wait for a go signal before carrying out the response. In a study that otherwise is quite similar to the present one, Elsner and Hommel (2004) used a rather complex S-R mapping, in which the location of an arrow indicated the hand to be moved, and the arrow itself indicated the direction of the movement. It is, 
thus, possible that the more complex or strong the intentional set necessary to carry out a stimulusbased action (i.e., the less reflex-like the action), the more likely that the actions are accompanied by ideomotor learning. However, notice that we did not observe any transfer effect in the delayed stimulus-based acquisition group, in which the actions of the acquisition phase-although stimulus based-were not very reflex-like. This shows that ideomotor learning is hampered whenever the action is selected with respect to some external demand, regardless of the immediacy of the execution of the action. In the light of this finding it seems unlikely that, for example, Elsner and Hommel (2004) observed ideomotor learning because-due to the complexity of the used $\mathrm{S}-\mathrm{R}$ mapping-the actions were less reflex-like.

Another possible reason for the divergent results concerns the test phase rather than the acquisition conditions. Priming research investigates the change in the speed or accuracy of processing of a probe event, as a result of prior experience with the same or a related prime event. It has been demonstrated that the more operation time the system needs during the probe event, the higher the likelihood to observe priming (e.g., Logan, 1988). Recently, Waszak and Hommel (in press) have shown that protracting the operation time of the probe event makes priming effects "appear" that otherwise go unnoticed. It is, thus, possible that it is the difference in overall operation time of the test phases that is responsible for the different results of the present study and the one from Elsner and Hommel (2004), for example. Probably due to the more complex response mapping, mean RTs in the test phase of Elsner and Hommel were between 400 and $500 \mathrm{~ms}$ and, thus, about $100 \mathrm{~ms}$ slower than those in the present study. Rather long mean RTs were also reported in the study of Hommel (2004). Ziessler and Nattkemper (2002) report mean RTs in the test phase that were even longer (between $700 \mathrm{~ms}$ and $950 \mathrm{~ms}$ ), probably also due to the more complex response mapping with eight stimuli and four responses. If the divergence of the results is indeed due to the test phases of the studies mentioned above being more sensitive to memory traces compiled during the acquisition phase, then the difference in results between the intention-based and the stimulus-based acquisition groups of the present study would rather be a question of degree. In that case intentionbased action selection would boost ideomotor learning to the point that it becomes measurable even if the test phase is rather insensitive to transfer effects. This would fit very well to certain findings in the literature-namely, that in humans the contribution of medial and lateral premotor cortices in intention-based and stimulus-based actions is also rather a matter of degree, with the medial premotor cortex being only more active in intention-based actions, but not exclusively (Deiber, Ibanez, Sadato, \& Hallett, 1996; Thut et al., 2000). This notion also makes sense from a theoretical point of view, since intention-based and stimulusbased actions are in the real world inextricably interwoven.

Binding operations as proposed above may be an integral process of selection-for-action and sensorimotor coordination. Binding together action codes, on the one hand, and codes of target stimuli or action effects, respectively, on the other hand, may result in the compilation of two different kinds of "dictionaries" instrumental to the control of action: The activity of the stimulusbased system may result in the compilation of a kind of stimulus-response dictionary containing rules about which motor routines action-relevant objects habitually require (sensorimotor learning). By contrast, the activity of the intention-based system may entail the compilation of a kind of action-effect dictionary containing rules about which action produces which effect (ideomotor learning). Both these kinds of memory trace may guide future behaviour when the participant has to act upon the same stimuli presented again or when the participant intends to produce a certain effect, respectively.

The notion that intention-based and stimulusbased actions are accompanied by two different kinds of binding mechanism is also 
supported by experiments on the perception of stimulus-response and response-effect events. In an experiment from Haggard, Aschersleben, Gehrke, and Prinz (2002a), participants had to judge the time when they pressed a key or when a tone was presented by indicating the position of a clock hand (Libet's clock procedure; Libet, Gleason, Wright, \& Pearl, 1983). Haggard et al. found that perceptual onset times of actions and their ensuing effects, on the one hand, and of stimuli and subsequent actions in response to them, on the other hand, attracted each other in time. Moreover, the temporal attraction between actions and their effects can be reversed when the tones are triggered by involuntary movements caused by transcranial magnetic stimulation (TMS) of the motor cortex (Haggard, Clark, \& Kalogeras, 2002b). The results from Haggard and colleagues are complemented by recent studies from Waszak et al. (2005b) and Keller et al. (2006). In both these studies, participants performed a temporal bisection task that involved making left or right keypresses at the midpoint between adjacent items in evenly timed sequences of visually presented pacing signals. In separate conditions participants either performed a choice response to a preceding stimulus (stimulusbased) or were free to select which of two keys to press to produce the next stimulus (intentionbased). In both studies intention-based actions were shifted in time towards their anticipated effects (the next stimulus), whereas stimulusbased actions were shifted towards their preceding stimulus. Waszak and colleagues considered these effects of the mode of movement on the actual timing of movements to be an analogue in the behavioural domain of the perceptual effects found by Haggard et al. (2002a). We suggest that the effects demonstrated in the present study, in turn, reflect the long-term consequences of the binding operations observed in the perceptual domain by Haggard et al. and in the timing domain by Waszak and colleagues.

Original manuscript received 18 April 2005

Accepted revision received 30 October 2006 First published online 21 February 2007

\section{REFERENCES}

Allport, D. A. (1987). Selection for action: Some behaviorial and neurophysiological considerations of attention and action. In H. Heuer \& A. F. Sanders (Eds.), Perspectives on perception and action (pp. 395-419). Hillsdale, NJ: Lawrence Erlbaum Associates, Inc.

Arcediano, F., Escobar, M., \& Miller, R. R. (2005). Bidirectional associations in humans and rats. Journal of Experimental Psychology: Animal Behavior Processes, 31, 301-318.

Astor-Jack, T., \& Haggard, P. (2005). Intention and reactivity. In G. W. Humphreys \& M. J. Riddoch (Eds.), Attention in action: Advances from cognitive neuroscience (pp. 109-130). Hove, UK: Psychology Press.

Beckers, T., De Houwer, J., \& Eelen, P. (2002). Automatic integration of non-perceptual action effect features: The case of the associative affective Simon effect. Psychological Research, 66, 166-173.

Cohen, J. (1988). Statistical power analysis for the social sciences (2nd ed.). Hillsdale, NJ: Lawrence Erlbaum Associates, Inc.

Deiber, M. P., Ibanez, V., Sadato, N., \& Hallett, M. (1996). Cerebral structures participating in motor preparation in humans: A positron emission tomography study. Journal of Neurophysiology, 75, 233-247.

Donders, F. C. (1969). On the speed of mental processes. (W. G. Koster, Trans.). Acta Psychologica, 30, 412-431. (Original work published 1868).

Elsner, B., \&Hommel, B. (2001). Effect anticipation and action control. Journal of Experimental Psychology: Human Perception E Performance, 27, 229-240.

Elsner, B., \& Hommel, B. (2004). Contiguity and contingency in the acquisition of action effects. Psychological Research, 68, 138-154.

Elsner, B., Hommel, B., Mentschel, C., Drzezga, A., Prinz, W., Conrad, B., et al. (2002). Linking actions and their perceivable consequences in the human brain. NeuroImage, 17, 364-372.

Exner, S. (1879). Physiologie der Grosshirnrinde [Physiology of the cerebral cortex]. In L. Hermann (Ed.), Handbuch der Physiologie (Vol. 2, Pt. 2, pp. 189-350). Leipzig, Germany: Vogel.

Faul, F., \& Erdfelder, E. (1992). GPOWER: A priori, post hoc, and compromise power analyses for MS-DOS [Computer program]. Bonn, Germany: Bonn University, Department of Psychology.

Fox, E. (1995). Negative priming from ignored distractors in visual selection: A review. Psychonomic Bulletin and Review, 2, 145-173. 
Haggard, P., Aschersleben, G., Gehrke, J., \& Prinz, W. (2002a). Action, binding and awareness. In W. Prinz \& B. Hommel (Eds.), Common mechanisms in perception and action: Attention and performance XIX (pp. 266-285). Oxford, UK: Oxford University Press.

Haggard, P., Clark, S., \& Kalogeras, J. (2002b). Voluntary action and conscious awareness. Nature Neuroscience, 5, 382-385.

Harless, E. (1861). Der Apparat des Willens. Zeitschrift für Philosophie und Philosophische Kritik, 38, 50-73.

Hoffmann, J., Sebald, A., \& Stoecker, C. (2001). Irrelevant response effects improve serial learning in serial reaction time tasks. Journal of Experimental Psychology: Learning, Memory, E Cognition, 27, 470-482.

Hommel, B. (1996). The cognitive representation of action: Automatic integration of perceived action effects. Psychological Research, 59, 176-186.

Hommel, B. (1998). Event files: Evidence for automatic integration of stimulus-response episodes. Visual Cognition, 5, 183-216.

Hommel, B. (2000). The prepared reflex: Automaticity and control in stimulus-response translation. In S. Monsell \& J. Driver (Eds.), Control of cognitive processes: Attention and performance XVIII (pp. 247273). Cambridge, MA: MIT Press.

Hommel, B. (2004). Coloring an action: Intending to produce color events eliminates the Stroop effect. Psychological Research, 68, 74-90.

Hommel, B., Pösse, B., \& Waszak, F. (2000). Contextualization in perception and action. Psychologica Belgica, 40, 227-245.

Keller, P., Wascher, E., Prinz, W., Waszak, F., Koch, I., \& Rosenbaum, D. (2006). Differences between intention-based and stimulus-based actions. Journal of Psychophysiology, 20, 9-20.

Kunde, W. (2004). Response priming by supraliminal and subliminal action effects. Psychological Research, 68, 91-96.

Kunde, W., Hoffmann, J., \& Zellmann, P. (2002). The impact of anticipated action effects on action planning. Acta Psychologica, 109, 137-155.

Lhermitte, F. (1983). "Utilisation behaviour" and its relation to lesions of the frontal lobes. Brain, 106, 237-255.

Libet, B., Gleason, C. A., Wright, E. W., \& Pearl, D. K. (1983). Time of conscious intention to act in relation to onset of cerebral activity (readiness potential). The unconscious initiation of a freely voluntary act. Brain, 106, 623-642.
Logan, G. D. (1988). Toward an instance theory of automatization. Psychological Review, 95, 492-527.

Logan, G. D., \& Etherton, J. L. (1994). What is learned during automatization? The role of attention in constructing an instance. Journal of Experimental Psychology: Learning, Memory, \& Cognition, 20, 1022-1050.

Lotze, R. H. (1852). Medizinische Psychologie oder die Physiologie der Seele [Medical psychology or physiology of the soul]. Leipzig, Germany: Weidmann'sche Buchhandlung.

Neill, W. T. (1997). Episodic retrieval in negative priming and repetition priming. Journal of Experimental Psychology: Learning, Memory, Eे Cognition, 23, 1291-1305.

Neill, W. T., \& Valdes, L. A. (1992). Persistence of negative priming: Steady state or decay? Journal of Experimental Psychology: Learning, Memory, E Cognition, 18, 565-576.

Norman, D., \& Shallice, T. (1986). Attention to action: Willed and automatic control of behavior. In R. Davidson, G. Schwartz, \& D. Shapiro (Eds.), Consciousness and self regulation: Advances in research and theory (Vol. 4, pp. 1-18). New York: Plenum.

Passingham, R. E., Toni, I., \& Rushworth, M. F. S. (2000). Specialisation within the prefrontal cortex: The ventral prefrontal cortex and associative learning. Experimental Brain Research, 133, 103-113.

Prinz, W. (1997). Perception and action planning. European Journal of Cognitive Psychology, 9, 129-154.

Shallice, T., Burgess, P. W., Schon, F., \& Baxton, D. M. (1989). The origins of utilization behavior. Brain, 112, 1587-1598.

Thut, G., Hauert, C. A., Viviani, P., Morand, S., Spinelli, L., Blanke, O., et al. (2000). Internally driven vs. externally cued movement selection: A study on the timing of brain activity. Cognitive Brain Research, 9, 261-269.

Toni, I., Rushworth, M. F. S., \& Passingham, R. E. (2001). Neural correlates of visuomotor associations: Spatial rules compared with arbitrary rules. Experimental Brain Research, 141, 359-369.

Waszak, F., \& Hommel, B. (in press). The costs and benefits of cross-task priming. Memory and Cognition.

Waszak, F., Hommel, B., \& Allport, A. (2003). Taskswitching and long-term priming: Role of episodic $\mathrm{S}-\mathrm{R}$ bindings in task-shift costs. Cognitive Psychology, 46, 361-413.

Waszak, F., Hommel, B., \& Allport, A. (2005a). Interaction of task readiness and automatic 
retrieval in task switching: Negative priming and competitor priming. Memory \& Cognition, 33, 595-610.

Waszak, F., Wascher, E., Keller, P., Koch, I., Aschersleben, G., Rosenbaum, D., et al. (2005b). Intention-based and stimulus-based mechanisms in action selection. Experimental Brain Research, 162, 346-356.

Ziessler, M. (1998). Response-effect learning as a major component of implicit serial learning. Journal of Experimental Psychology: Learning, Memory, E० Cognition, 24, 962-978.
Ziessler, M., \& Nattkemper, D. (2001). Learning of event sequences is based on response effect learning: Further evidence from a serial reaction task. Journal of Experimental Psychology: Learning, Memory, E० Cognition, 27, 595-613.

Ziessler, M., \& Nattkemper, D. (2002). Effect anticipation in action planning: Anticipative learning of action effects. In W. Prinz \& B. Hommel (Eds.), Common mechanisms in perception and action: Attention and performance XIX (pp. 645-672). Oxford, UK: Oxford University Press. 